\title{
Noble Metal High Entropy Alloys
}

\author{
Sungwoo Sohn ${ }^{1,2}$, Yanhui Liu ${ }^{1,2}$, Jingbei Liu ${ }^{1,2}$, Pan Gong ${ }^{3}$, Silke Prades-Rodel ${ }^{4}$, Andreas \\ Blatter $^{4}$, B. Ellen Scanley ${ }^{2,5}$, Christine C. Broadbridge ${ }^{2,5}$, Jan Schroers ${ }^{1,2}$ \\ ${ }^{1}$ Department of Mechanical Engineering and Materials Science, Yale University, New Haven, \\ CT 06511, USA \\ ${ }^{2}$ Center for Research on Interface Structures and Phenomena, Yale University, New Haven, CT \\ 06511, USA \\ ${ }^{3}$ State Key Laboratory of Materials Processing and Die \& Mould Technology, Huazhong \\ University of Science and Technology, 1037 Luoyu Road, Wuhan 430074, Hubei, China \\ ${ }^{4}$ PX Holding SA, 2304 La Chaux-de-Fonds, Switzerland \\ ${ }^{5}$ Department of Physics, Southern Connecticut State University, New Haven, CT 06515, USA
}




\begin{abstract}
A series of noble metal high entropy alloys with up to six constituent elements has been produced by casting. PtPdRhIrCuNi forms single-phase face-centered cubic solid solution, and its stability is confirmed by annealing experiments. This alloy deforms homogeneously to $\sim 30 \%$ to a high ultimate compression strength of $1839 \mathrm{MPa}$. We discuss rules for the formation of single-phase solid solution.
\end{abstract}

Keywords : High entropy alloys; FCC structure; solid solution; Compressive property; Phase Stability 
In contrast to conventional alloys, which are typically composed of only one or two principal elements, high entropy alloys (HEAs), consist of five or more elements with (near) equiatomic concentration, which form single-phase solid solution (SPSS) [1-5]. This design strategy greatly increases the number of potential technologically relevant alloys $[6,7]$. The mere combination of practical elements suggests vast numbers of potential HEAs. However, prediction of HEAs out of such vast numbers of potential alloys has been proven to be difficult $[6,7]$. Predicting stable phases require the knowledge and comparison of the Gibbs free energy of all possible phases [8]. However, this is beyond today's computing power and hence assumptions for $\Delta H_{\text {mix }}$, and $\Delta S_{\text {mix }}$ have been suggested [9-12] based on regular mixing behavior $[9,10,13-16]$. The entropy term has been estimated assuming random configuration contribution at equiatomic composition to $S_{\mathrm{c}}=k \ln n$ ( $n$ : number of components) [1]. In the attempt to estimate the enthalpic contribution, pairwise $\Delta H_{\text {mix }}$ were considered $[9,10,14,16,17]$. The effect of mixing many elements is estimated through $\Delta H_{\text {mix }}=\sum_{i=1, j>i}^{n} 4 \Delta H c_{i} c_{j}$ which is the weighted average of all $\Delta H$, the pairwise enthalpy of mixing. For SPSS to form, it has been suggested from empirical considerations $[9,10]$ that this averaged $\Delta H_{\text {mix }}$ should be in the range from -11.6 to $3.2 \mathrm{~kJ} / \mathrm{mol}$ [9]. However, deviation from this rule has been reported [9, 11, 15, 17], suggesting oversimplifications in the estimation of the enthalpic contribution. In addition to thermodynamics, geometric consideration similar to Hume-Rothery has been identified to be crucial in phase formation of HEAs [18, 19]. For the formation of solid solutions, it was suggested that the atomic size difference, $\delta,\left(\delta=\sqrt{\sum_{i}^{n} c_{i}\left(1-r_{i} / \sum_{i}^{n} c_{i} r_{i}\right)^{2}} \times 100\right.$, where $r_{i}$ is the atomic radius of the $i^{\text {th }}$ atom) should be smaller than $6.6 \%$ [9].

Considering both thermodynamic and geometrical factors affecting phase formation, we report new HEA systems consisting of noble metals. We found for the noble metals HEAs that, 
in addition to the weighted average $\Delta H_{\text {mix }}$, enthalpy of mixing for individual pairs plays a vital role in phase formation.

We considered $\mathrm{Au}, \mathrm{Pd}, \mathrm{Ag}, \mathrm{Pt}, \mathrm{Rh}, \mathrm{Ir}, \mathrm{Cu}$ and $\mathrm{Ni}$ for potential alloy constituents. All of the elements are faced-centered cubic (FCC) forming elements as listed in Table 1. Binary (AuPd), ternary (AuPdAg), quaternary (AuPdAgPt), quinary (AuPdAgPtCu), and senary (AuPdAgPtCuNi and PdPtRhIrCuNi) alloys at equiatomic composition of approximately $10 \mathrm{~g}$ were produced by arc melting of high purity metals. The crystal structure and microstructure of the alloys were studied using X-ray diffraction (XRD) (Rigaku, SmartLab) and scanning electron microscopy (SEM) (Zeiss, Sigma VP) with backscatter electron (BSE). Chemical analysis was performed by using energy-dispersive X-ray spectrum (EDS) attached to SEM. Specimens of dimension of $2 \times 2 \times 4 \mathrm{~mm}$ for compression tests were electric-discharged machined from $3 \mathrm{~mm}$ cast samples. Compression tests were conducted using an Instron mechanical testing machine at a constant strain rate of $10^{-4} \mathrm{~s}^{-1}$.

XRD patterns of the as-cast AuPd, AuPdAg, AuPdAgPt, AuPdAgPtCu, AuPdAgPtCuNi, and PdPtRhIrCuNi alloys are shown in Fig. 1. All peaks have been identified to be solid solutions of FCC structure (space group: Fm-3m). Formation of FCC phases can be expected because all constituent elements are FCC structures, corresponding solid solutions in every possible binary combination appear to form FCC structures upon solidification at equiatomic composition, and most of the constituent elements have similar physicochemical and thermodynamic parameters (Table 1 and Table S1), such as atomic radii (1.25 1.39 ̊́), Pauling electronegativity values (1.90 2.54) and valence electron numbers (9 11). Furthermore, for all considered alloys, $\delta$ is smaller than $6.6 \%$ and $\Delta H_{\text {mix }}$ is within the range of $-11.6 \sim 3.2 \mathrm{~kJ} / \mathrm{mol}$ (Table 1). 
The XRD patterns exhibit, only one set of FCC diffraction peaks for AuPd, AuPdAg, and AuPdAgPt, suggesting that these alloys form SPSS. However, a second phase of FCC structure forms when $\mathrm{Cu}$ is added into $\mathrm{AuPdAgPt}$ as demonstrated by the additional set of FCC diffraction peaks in AuPdAgPtCu. Although both $\delta$ and $\Delta H_{\text {mix }}$ remain in the solid solution forming range after $\mathrm{Cu}$ addition, pairs of $\mathrm{Pt}-\mathrm{Cu}$ and $\mathrm{Pd}-\mathrm{Cu}$ have higher negative enthalpy of mixing compared to the other pairs (Table 2). This indicates that a significant deviation of individual pairs from the average can promote formation of secondary phases. In the attempt to cancel the effect of large negative $\Delta H$ from the $\mathrm{Pt}-\mathrm{Cu}$ and $\mathrm{Pd}-\mathrm{Cu}$ pairs, we added $\mathrm{Ni}$ which has a positive $\Delta H$ with $\mathrm{Ag}$ and $\mathrm{Au}$ (Table 2). This approach however is not successful, a two phase microstructure remains in AuPdAgPtCuNi (Fig. 1). This suggest that a mere consideration of average $\Delta H_{\text {mix }}$ may be insufficient in predicting SPSS, Instead we considered introducing pairs of moderately positive $\Delta H$ by substituting Au and Ag with Rh and Ir (Table 2). As can be seen in Fig. 1, only one FCC phase is present in PdPtRhIrCuNi.

Microstructures observed by SEM-BSE from the central parts of the as-cast samples are shown in Fig. 2(a) and (b) for AuPdAgPtCuNi and PdPtRhIrCuNi, respectively. The AuPdAgPtCuNi alloy shows a microstructure consisting of primary dendrite phases embedded in matrix phases of darker contrast. With length scale ranging from 5 to $10 \mu \mathrm{m}$, the dendritic phase is estimated to have a volume fraction about 10 to $20 \%$. These two phases correspond to the two FCC phases in XRD patterns. On the other hand, the single solid solution forming PdPtRhIrCuNi exhibits a completely dendritic microstructure, as shown in Fig. 2(b). The average size of primary arm is about $5 \mu \mathrm{m}$. Chemical analyses indicate that the dendrite is $\mathrm{Rh}$ and Ir rich, e.g. $\mathrm{Pd}_{8} \mathrm{Pt}_{13} \mathrm{Rh}_{26} \mathrm{Ir}_{34} \mathrm{Cu}_{6} \mathrm{Ni}_{13}$. The interdendritic region exhibit a varying composition with up to $\mathrm{Pd}_{20} \mathrm{Pt}_{21} \mathrm{Rh}_{13} \operatorname{Ir}_{7} \mathrm{Cu}_{18} \mathrm{Ni}_{21}$. 
In general, there are two possible origins of the compositional variations in the microstructure; non-equilibrium solidification [2] and phase separation [20]. Under a higher diffusive condition, composition gradient should decrease to form a homogeneous SPSS for nonequilibrium solidification and increase for phase separation due to difference in thermodynamic driving force, as schematically illustrated in Fig. 3(a). In order to determine which scenario is present in PdPtRhIrCuNi, we conducted annealing experiments both below and above $T_{\mathrm{m}}$ of the alloy, with the evolution of the element distribution revealed by EDS mapping (Fig. 3(b)-(e)). In the as-cast state (Fig. 3(b)), the distribution of the elements is non-uniform with the dendritic region rich in $\mathrm{Rh}$ and $\mathrm{Ir}$ and poor in $\mathrm{Pd}$ and $\mathrm{Cu}$. When being annealing at $1000{ }^{\circ} \mathrm{C}$ (Fig. 3(d)), element distribution becomes more uniform. Further increasing annealing temperature to 1200 ${ }^{\circ} \mathrm{C}$ results in further reduction of composition variations. However, increasing annealing temperature to $1400{ }^{\circ} \mathrm{C}$ (Fig. 3(e)) which is above $T_{\mathrm{s}}$ of the alloy, composition variation increases rapidly. To quantify the compositional difference between dendritic and interdendritic regions, we use $\Delta C_{\text {sum }}=\sum\left|C_{i \text {,bright }} C_{i, \text { dark }}\right|$ as an indicator, where $i$ represent the constituent elements so that less difference leads to smaller value of $\Delta C_{\text {sum. }}$ Fig. 3(f) shows the change of $\Delta C_{\text {sum }}$ with annealing temperature. The concentration changes for each element can be found in Supplementary Materials. As shown in Fig. 3(b), $\Delta C_{\text {sum }}$ decreases with increasing annealing temperature from $1000{ }^{\circ} \mathrm{C}$ to $1200{ }^{\circ} \mathrm{C}$. At $1400{ }^{\circ} \mathrm{C}>T_{\mathrm{s}}, \Delta C_{\text {sum }}$ increases. The changes of $\Delta C_{\text {sum }}$ with annealing temperature suggest that the compositional variations in as-cast PdPtRhIrCuNi originate from non-equilibrium solidification and the alloy evolves into a homogenous in composition SPSS phase. To confirm if the alloy indeed develops towards a uniform composition FCC SPSS phase, we conducted another set of annealing experiments at constant temperature $\left(1200{ }^{\circ} \mathrm{C}\right)$ for various annealing conditions from $2 \mathrm{hrs}$ to $200 \mathrm{hrs}$, which can be 
shown in Supplementary Materials. We found that the volume fraction of the bright phase gradually decreases as a function of time [21]. Our results indicate that the microstructure develops towards a homogeneous FCC SPSS phase when approaching equilibrium.

HEAs have been reported to possess a combination of high strength and ductility $[4,22]$. To evaluate the mechanical property of the PdPtRhIrCuNi, we performed compression tests. As shown in Fig. 4(a), the yield strength, $\sigma_{y}$, of PdPtRhIrCuNi is $527 \mathrm{MPa}$, while the maximum strength, $\sigma_{\mathrm{m}}$, is $1839 \mathrm{MPa}$. In comparison with previously reported HEAs that form FCC SPSS, the strength of PdPtRhIrCuNi is higher because of the "Cocktail effect" [3], originating especially from the high strength of Rh and Ir. According the stress-strain curve shown in Fig. 4(a), the strain to failure is $32.4 \%$, a value typical for FCC structured HEAs [23]. Fracture appears to occur along the maximum shear stress (Fig. 4(b)). As marked by arrows, numerous slip bands can be observed on sample surface, indicating the homogeneous deformation upon loading. In addition, the data show a dramatic solid-solution-like strengthening mechanism for the single-phase FCC alloy (PdPtRhIrCuNi) which is not present in the two phases alloy (AuPdAgPtCuNi).

In summary, we designed a noble metal HEA, PdPtRhIrCuNi. Annealing experiments reveal that the stability of a single-phase FCC solid solution. Mechanical characterization revealed a high ultimate compression strength of $1839 \mathrm{MPa}$ and large ductility in excess of $30 \%$, suggesting the operation of a solid-solution-like strengthening mechanism. Comparisons of similar senary alloys revealed that average values for heat of mixing are insufficient in indicating the formation of single-phase solid solutions. By comparing two senary alloys that follow the phase selection criteria currently used for the design of HEAs, PtPdRhIrCuNi which forms a SPSS and AuPdAgPtCuNi which exhibits a two phase mixture, we found that the phase selection 
is not fully predictable. We attribute the formation of dual phases in AuPdAgPtCuNi to the large pairwise heat of mixing.

\section{Acknowledgements}

This work was supported by the National Science Foundation under DMREF Grants No. DMR-

1436268. Experiments were performed in part at the Materials Research Science and

Engineering Center at Yale University (supported by NSF under Award No. DMR-1119826). 


\section{References}

1. Yeh, J.W., et al., Nanostructured high-entropy alloys with multiple principal elements: Novel alloy design concepts and outcomes. Advanced Engineering Materials, 2004. 6(5): p. 299-303.

2. Cantor, B., et al., Microstructural development in equiatomic multicomponent alloys. Materials Science and Engineering a-Structural Materials Properties Microstructure and Processing, 2004. 375: p. 213-218.

3. Yeh, J.W., Physical Metallurgy of High-Entropy Alloys. JOM, 2015. 67(10): p. 22542261.

4. Zhang, Y., et al., Microstructures and properties of high-entropy alloys. Progress in Materials Science, 2014. 61: p. 1-93.

5. Lu, Z.P., et al., An assessment on the future development of high-entropy alloys: Summary from a recent workshop. Intermetallics, 2015. 66: p. 67-76.

6. Senkov, O.N., et al., Accelerated exploration of multi-principal element alloys with solid solution phases. Nature Communications, 2015. 6.

7. Miracle, D.B., et al., Exploration and Development of High Entropy Alloys for Structural Applications. Entropy, 2014. 16(1): p. 494-525.

8. Guo, S., Phase selection rules for cast high entropy alloys: an overview. Materials Science and Technology, 2015. 31(10): p. 1223-1230.

9. Guo, S., et al., More than entropy in high-entropy alloys: Forming solid solutions or amorphous phase. Intermetallics, 2013. 41: p. 96-103.

10. Zhang, Y., et al., Solid-solution phase formation rules for multi-component alloys. Advanced Engineering Materials, 2008. 10(6): p. 534-538.

11. Senkov, O.N. and D.B. Miracle, A new thermodynamic parameter to predict formation of solid solution or intermetallic phases in high entropy alloys. Journal of Alloys and Compounds, 2016. 658: p. 603-607.

12. Troparevsky, M.C., et al., Criteria for Predicting the Formation of Single-Phase HighEntropy Alloys. Physical Review X, 2015. 5(1).

13. Ye, Y.F., et al., Design of high entropy alloys: A single-parameter thermodynamic rule. Scripta Materialia, 2015. 104: p. 53-55. 
14. King, D.J.M., et al., Predicting the formation and stability of single phase high-entropy alloys. Acta Materialia, 2016. 104: p. 172-179.

15. Otto, F., et al., Relative effects of enthalpy and entropy on the phase stability of equiatomic high-entropy alloys. Acta Materialia, 2013. 61(7): p. 2628-2638.

16. Yang, X. and Y. Zhang, Prediction of high-entropy stabilized solid-solution in multicomponent alloys. Materials Chemistry and Physics, 2012. 132(2-3): p. 233-238.

17. Zhang, Y., et al., Guidelines in predicting phase formation of high-entropy alloys. Mrs Communications, 2014. 4(2): p. 57-62.

18. Wang, Z.J., et al., Atomic-size effect and solid solubility of multicomponent alloys. Scripta Materialia, 2015. 94: p. 28-31.

19. Guo, S., et al., Solid solutioning in equiatomic alloys: Limit set by topological instability. Journal of Alloys and Compounds, 2014. 583: p. 410-413.

20. Cahn J.W., On spinodal decomposition. Acta Metallurgica, 1961. 9: p. 795-801.

21. Tsai, K.Y., M.H. Tsai, and J.W. Yeh, Sluggish diffusion in Co-Cr-Fe-Mn-Ni high-entropy alloys. Acta Materialia, 2013. 61(13): p. 4887-4897.

22. Gludovatz, B., et al., A fracture-resistant high-entropy alloy for cryogenic applications. Science, 2014. 345(6201): p. 1153-1158.

23. Tong, C.J., et al., Microstructure characterization of AlxCoCrCuFeNi high-entropy alloy system with multiprincipal elements. Metallurgical and Materials Transactions a-Physical Metallurgy and Materials Science, 2005. 36A(4): p. 881-893. 


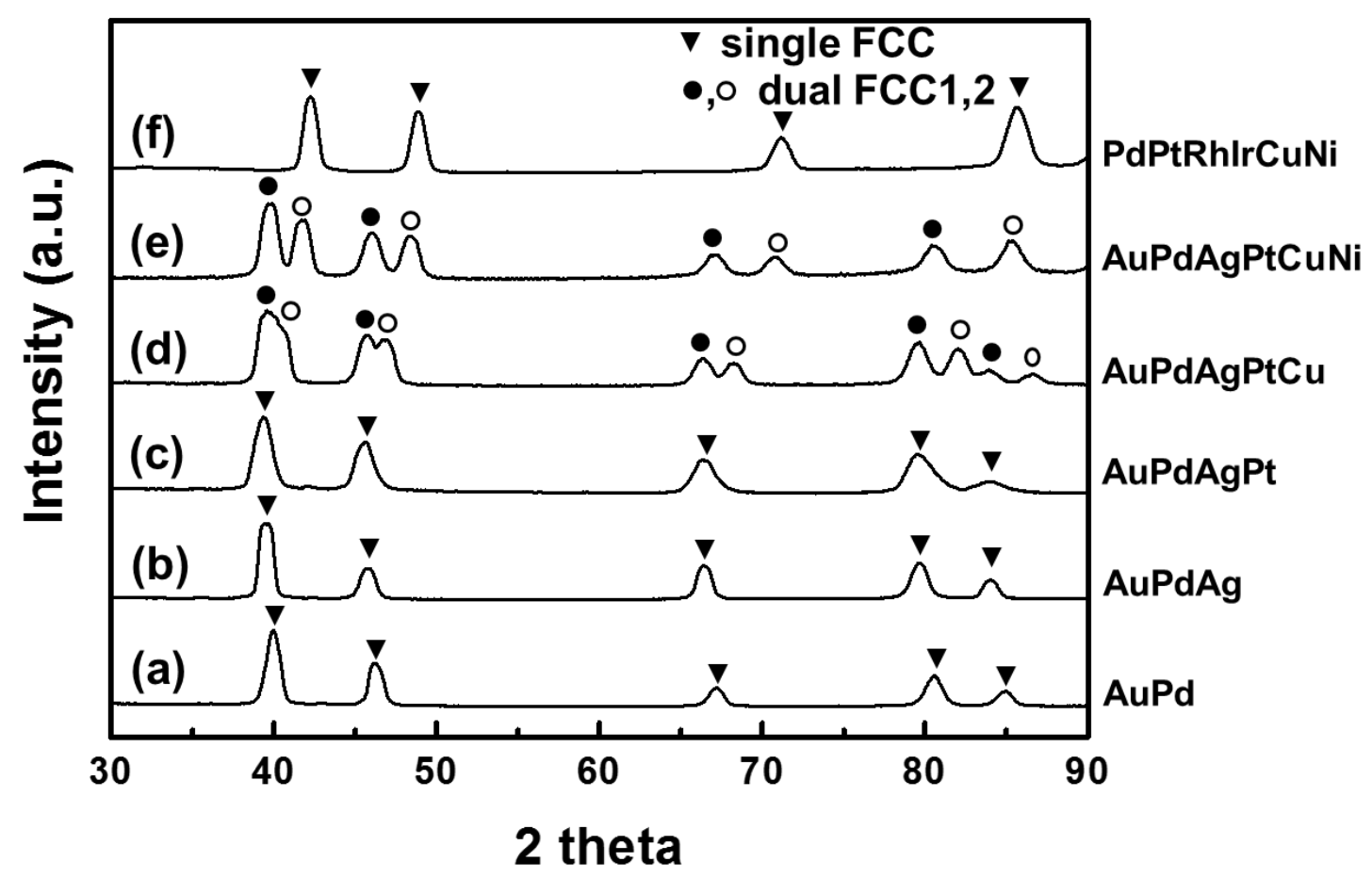

Figure 1. X-ray diffraction patterns of (a) AuPd, (b) AuPdAg, (c) AuPdAgPt, (d) AuPdAgPtCu, (e) AuPdAgPtCuNi, and (f) PdPtRhIrCuNi. 

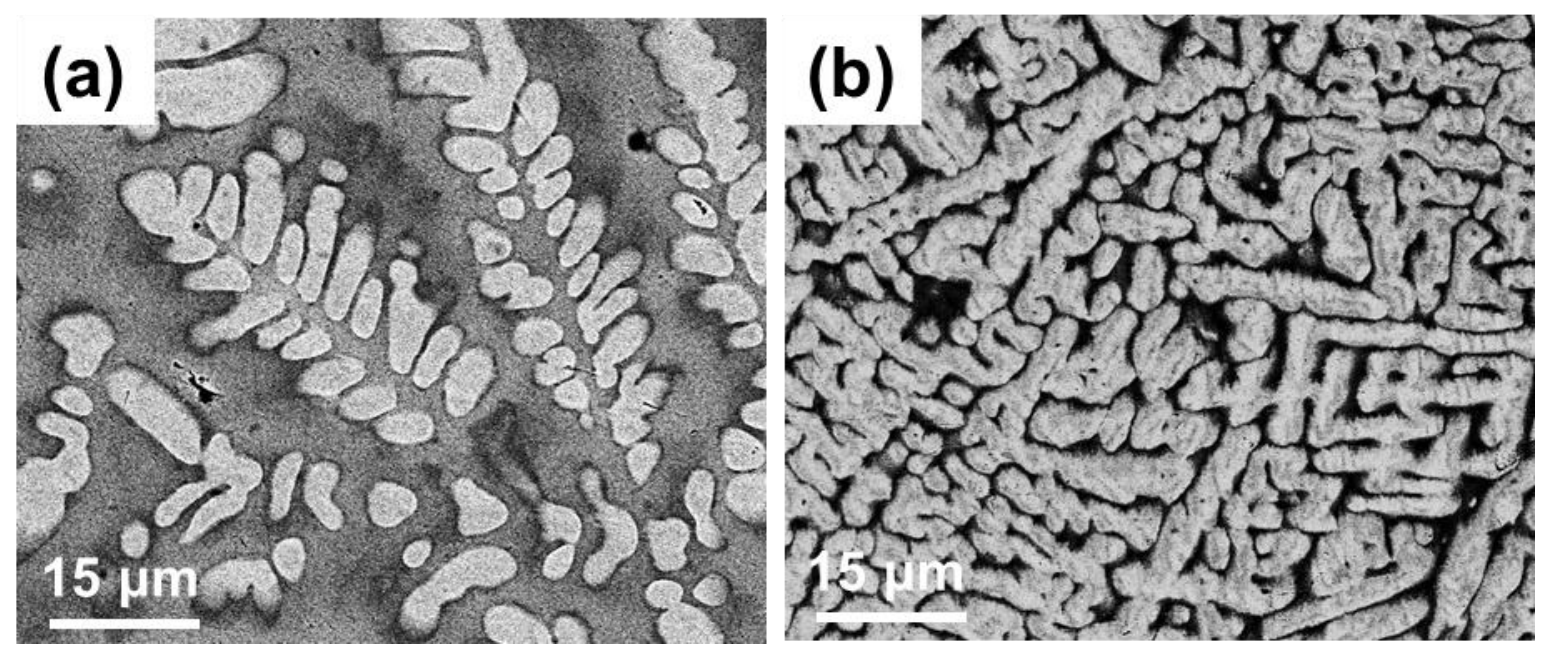

Figure 2. SEM-BSE morphologies at the central regions of the as-cast samples. (a)AuPdAgPtCuNi, and (b)PdPtRhIrCuNi. 


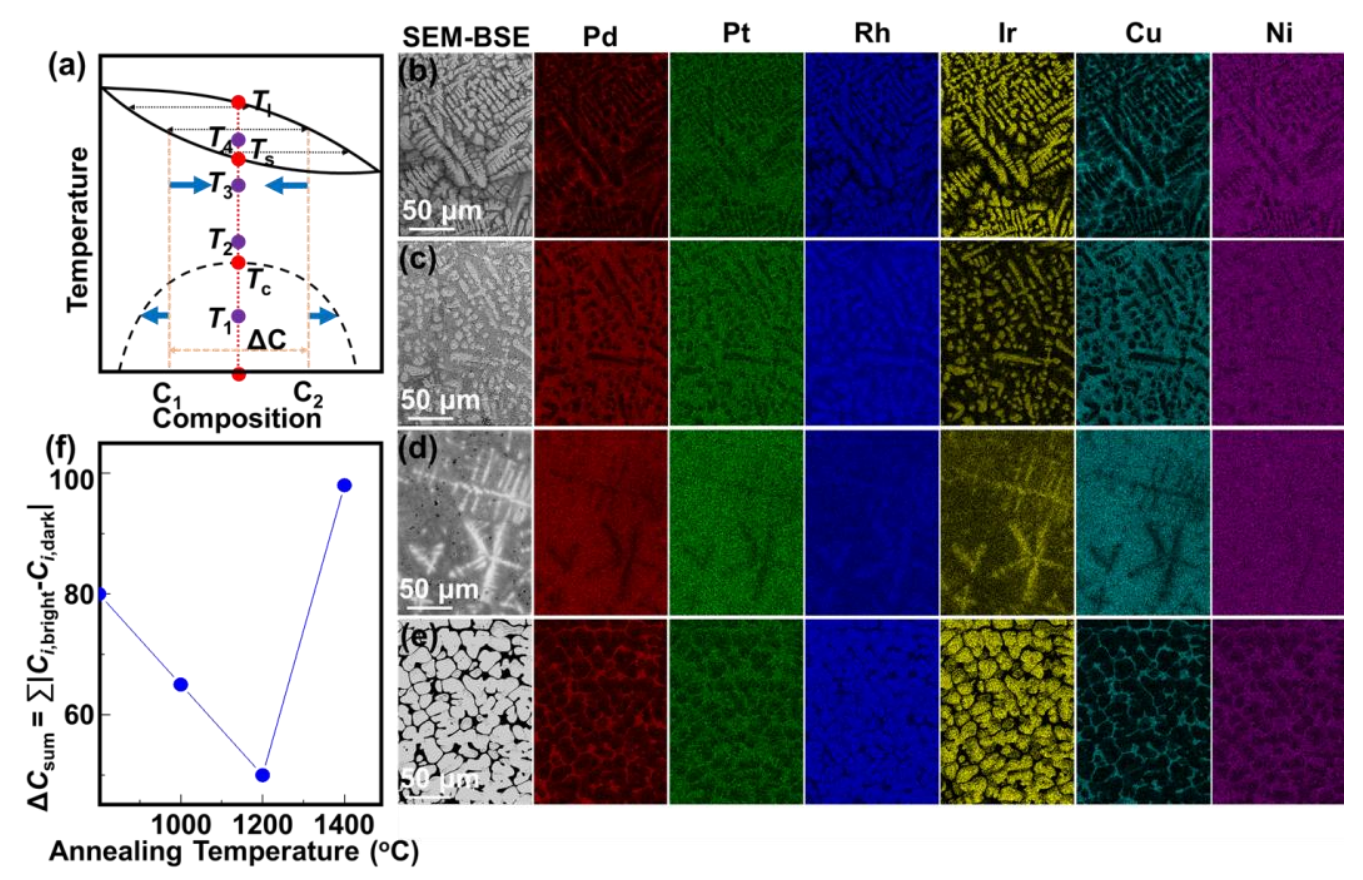

Figure 3. (a) Situations that can result into compositional variations within the microstructure of alloys that are fabricated by non-equilibrium cooling. Decrease in total compositional difference within the microstructure upon subsequent annealing temperature below the solidus temperature, e.g., $T_{3}$, suggest a stable single solid solution region. . Increasing $\Delta \mathrm{C}$ with annealing is indicative of a spinodal in the system, e.g., at $T_{1}$ equilibrium situation comprises of two-phase mixture. (b)(e) Elemental distributions obtained by EDS mapping for PdPtRhIrCuNi alloy: (b) as-cast, (c)-(e) annealed at $1000{ }^{\circ} \mathrm{C}, 1200{ }^{\circ} \mathrm{C}$, and $1400{ }^{\circ} \mathrm{C}$ for 2 hrs. (f) $\Delta C_{\text {sum }}=\sum\left|C_{i \text {,bright }} C_{i, \text { dark }}\right|$, an indicator of compositional difference between dendritic and interdendritic regions, as function of annealing temperature for of PdPtRhIrCuNi alloy. Detailed compositions of the two regions after each annealing can be found in Table S4. 


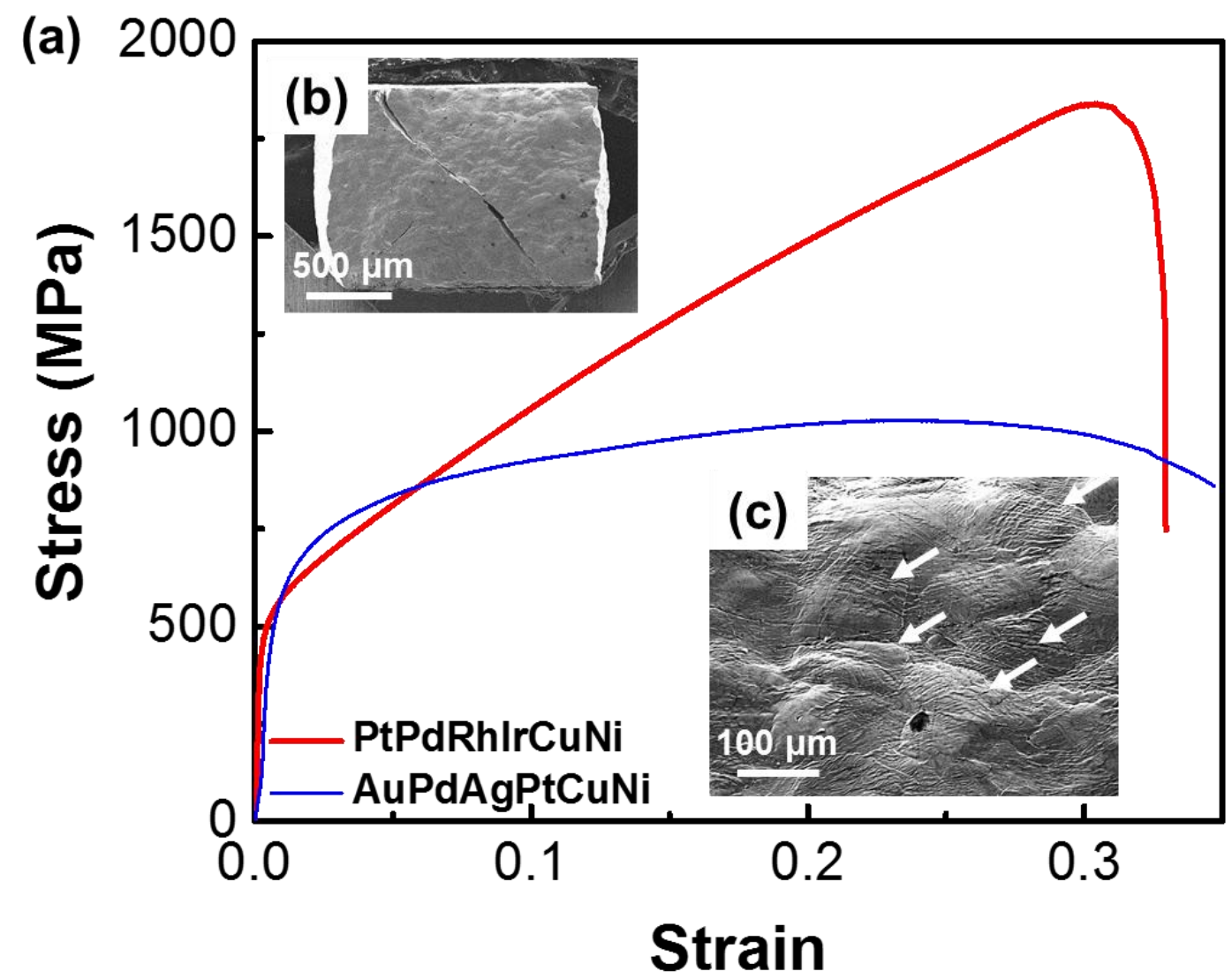

Figure 4. (a) Compressive stress-strain curves for the PdPtRhIrCuNi alloy (red) and AuPdAgPtCuNi alloy (blue). (b)-(c) SEM images of the sample surface after compression test. 
Table 1. Crystal structure, lattice parameter, $a$, atomic radius, $r$, and melting temperature, $T_{\mathrm{m}}$, for considered elements.

\begin{tabular}{ccccccccc}
\hline & Au & Pd & Ag & Pt & Rh & Ir & Cu & Ni \\
\hline structure & FCC & FCC & FCC & FCC & FCC & FCC & FCC & FCC \\
$a, \AA$ & 4.078 & 3.891 & 4.085 & 3.924 & 3.803 & 3.839 & 3.615 & 3.524 \\
$r, \AA$ & 1.39 & 1.34 & 1.37 & 1.35 & 1.33 & 1.35 & 1.25 & 1.25 \\
$T_{\mathrm{m}},{ }^{\circ} \mathrm{C}$ & 1064 & 1555 & 962 & 1768 & 1964 & 2466 & 1085 & 1455 \\
\hline
\end{tabular}


Table 2. List of atomic-size difference, $\delta$, enthalpy of mixing, $\Delta H_{\mathrm{mix}}$, highest positive enthalpy of mixing $\Delta H_{\max }$, and highest negative $\Delta H_{\min }$.

\begin{tabular}{ccccc}
\hline alloys & $\boldsymbol{\delta}[\%]$ & $\begin{array}{c}\Delta \boldsymbol{H}_{\text {mix }} \\
{[\mathbf{k J} / \mathbf{m o l}]}\end{array}$ & $\begin{array}{c}\Delta \boldsymbol{H}_{\text {max }} \\
{[\mathbf{k J} / \mathbf{m o l}]}\end{array}$ & $\begin{array}{c}\Delta \boldsymbol{H}_{\text {min }} \\
{[\mathbf{k J} / \mathbf{m o l}]}\end{array}$ \\
\hline AuPd & 2.49 & 0 & 0 & 0 \\
AuPdAg & 2.33 & -5.78 & 0 & -7 \\
AuPdAgPt & 2.19 & -2.00 & 4 & -7 \\
AuPdAgPtCu & 4.25 & -6.56 & 4 & -14 \\
AuPdAgPtCuNi & 5.39 & -2.22 & 15 & -14 \\
PdPtRhIrCuNi & 3.71 & -2.56 & 6 & -14 \\
\hline
\end{tabular}




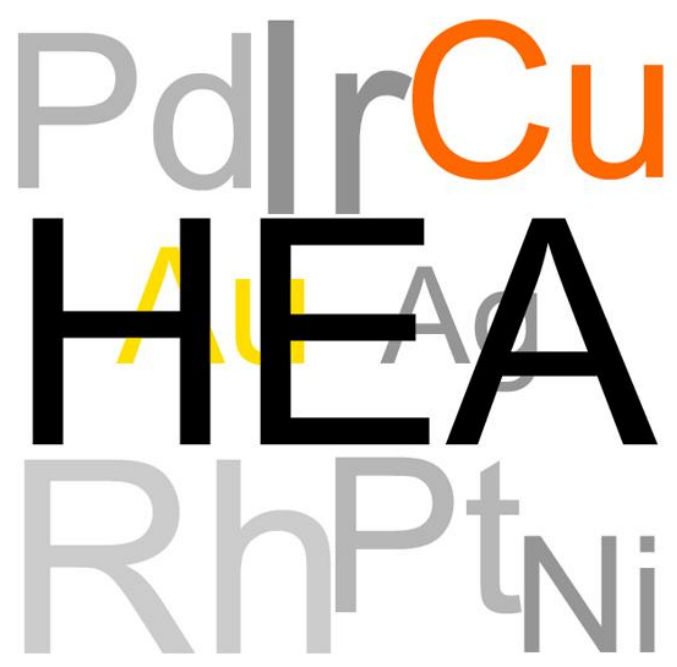

"Noble Metal high Entropy Alloys"

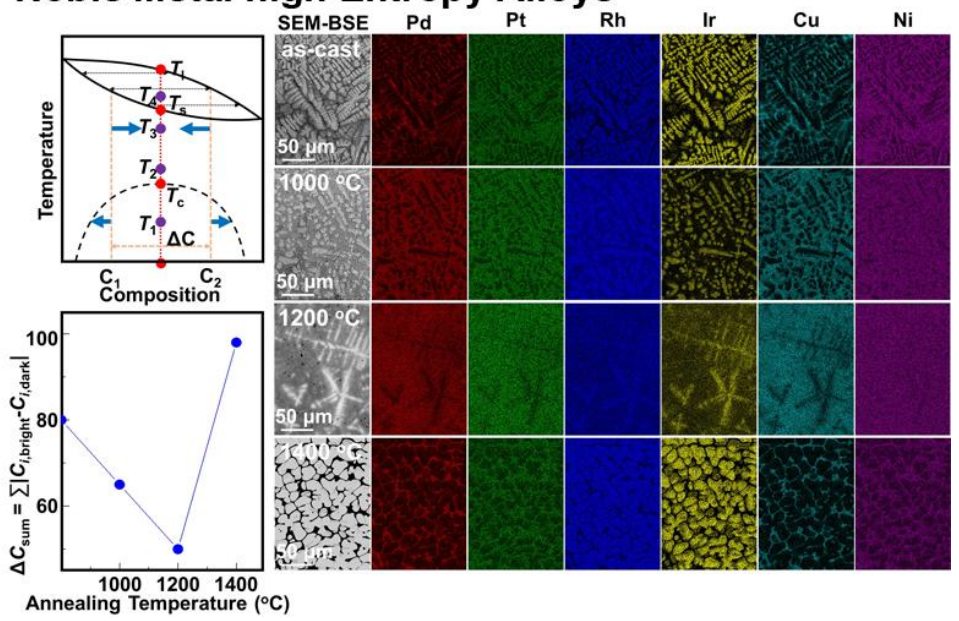

Graphical abstract 